\title{
HIBRIDISMO Y FRONTERA EN LA LITERATURA MARGINAL CONTEMPORÁNEA DE ARGENTINA Y BRASIL
}

\author{
Hibridization and border in contemporary marginal literature of Argentina and Brazil
}

\author{
Gabriela Leighton*
}

Amanda Leal**

\begin{abstract}
RESUMEN
Con sus similitudes y diferencias, la emergencia de una literatura marginal contemporánea en Brasil y en Argentina es un fenómeno social que se radica en las denominadas favelas y en los barrios o villas. En ese sentido, sus narrativas describen el contexto social vivido en los barrios periféricos, posibilitando que diversos "Yoes" que estaban silenciados "hablen" en un campo literario que era hasta entonces lugar de privilegio y de "alta cultura". Dicha literatura es muchas veces interpretada desde una perspectiva legitimista o relativista. Sin embargo, esas claves analíticas impiden identificar las especificidades, las desviaciones y los quiebres narrativos presentes en el emergente campo literario de la literatura marginal, o "literatura villera". Palabras clave: literatura comparada, literatura marginal, géneros.
\end{abstract}

\begin{abstract}
With many things alike and some differences, the emergence of a contemporary marginal literature in Brazil and in Argentina is a social phenomenon located in the so called "favelas" and the "barrios" or "villas" (deprived neighbourhoods). Therefore, those narratives describe the social context of these peripheral "barrios", giving voice to diverse "Me" within a literary field that was before a privilege of the "high culture". Such literature is many times interpreted from a legitimist or relativist point of view. Nevertheless, those analytical keys prevent us from identifying the specific features, deviations and narrative breakages that are present in the emerging literary field of this marginal literature or "Villera Literature".
\end{abstract}

Key Words: comparative literature, marginal literature, genre. 


\section{Nuevos vientos soplan en el campo literario latinoamericano}

Desde villas y favelas irrumpe desbordante, sobresaliente, la literatura marginal contemporánea de Argentina y Brasil, un nuevo campo literario que refleja la realidad social de los barrios al tiempo que recupera sus voces silenciadas. Esta literatura de los bordes interpela de forma activa a toda la sociedad y representan un ataque, una entrada brusca.

El objetivo del presente artículo es establecer un diálogo transdisciplinar entre la sociología de la cultura y los estudios literarios que permita interpretar los aspectos constitutivos de las obras citadas, en particular la frontera como el lugar de constitución del texto, y la disrupción lingüística como respuesta a la lengua canónica y sus formas.

El diálogo entre ambas disciplinas es clave para interpretar la literatura marginal contemporánea, para no caer en una cierta miopía frecuentemente presente entre los científicos sociales que consideran poseer el monopolio de las representaciones sociales. Así, las herramientas propias de su disciplina, por ejemplo, de la antropología o la sociología, serían las únicas correctas para describir o investigar a una realidad social. Sin embargo, como bien nos recuerda Howard Becker (2015), esa es apenas una de las formas de analizarla. Observando el modo en el que colegas académicos de otras disciplinas, y también personas que no están vinculadas con la academia, representan e interpretan a la sociedad, nos permite ampliar el alcance de nuestra mirada que, si nos encerráramos en los métodos de nuestra profesión no identificaríamos.

Para cumplir con el objetivo que nos hemos propuesto sin caer en trampas, no podemos utilizar nociones legitimistas o relativistas que manifiesten una mirada sesgada que -basada en las condiciones en que es producida- considere imposible a la literatura marginal contemporánea o bien la estigmatice como maravillosa.

Por eso, para develar sus características, debemos interpretarla desde una perspectiva latinoamericana. Así, daremos cuenta de los elementos específicos de esta literatura de frontera con sus narrativas, sus quiebres estructurales, su relación conflictiva con el campo literario y las condiciones sociales que posibilitan su desarrollo en Argentina y Brasil.

\subsection{Una mirada desde la Sociología de la Cultura}

\subsubsection{La Frontera}

La frontera posee dos connotaciones en este trabajo. La primera es topológica, ya que estas narrativas se desarrollan en barrios localizados en los márgenes de las grandes urbes de Argentina y de Brasil.

La segunda está relacionada con una mirada estética. La literatura marginal contemporánea de Argentina y Brasil visibiliza representaciones sociales vivenciadas en estos barrios que son considerados desde una perspectiva legitimista como lugares despreciables, de extrema violencia y pobreza y yermos para producir literatura alguna.

La literatura marginal contemporánea de Argentina y Brasil interpela al resto de la sociedad que transborda sus fronteras. Estos autores establecen un puente entre realidades distintas, en un ir y venir que transciende a los lugares que -siguiendo a Bourdieu- le son normalmente asignados a los distintos actores sociales según su posición social, la cual deriva de su condición de clase (Bourdieu, 1988: 38).

\subsubsection{El campo artístico}

Pierre Bourdieu argumenta que los bienes culturales solamente adquieren esa denominación cuando son poseedores de "la creencia" como valor agregado. Ahora bien, ¿qué es el campo artístico? ¿Quién o qué define su legitimidad, el reconocimiento de un escritor, de una novela?

En su clásica definición de campo, el sociólogo francés destaca que toda representación social $-\mathrm{y}$ en nuestro caso específico el mundo de la literatura- obedece a leyes sociales 
que le son específicas. Es decir, cada campo posee una autonomía, un conjunto de reglas propias que pueden o no circular en otros campos. La formación de cada uno de ellos es acompañada por una acumulación de saberes, técnicas y procedimientos que los hace genuinos $\mathrm{y}$, consecuentemente, marcados por luchas simbólicas. Es por ese carácter irreversible que los campos son reacios a cualquier forma de variación (Bourdieu, 1998: 38).

Las reglas que constituyen cada campo generan un rechazo a cualquier nuevo elemento que desafíe su propio nomos. Además, hay dos características complementarias sustanciales que debemos tener en consideración para descifrar las disputas emergentes en el campo literario. En primer lugar, es primordial identificar cuáles son las posiciones que las personas pueden ocupar en un campo determinado. El nivel de esas posibilidades, por ejemplo, el que un escritor sea reconocido como tal, dependerá del capital simbólico garantizador de reconocimiento que este posea. Así, ese capital está distribuido de forma desigual entre los diferentes escritores. Consecuentemente, el lugar que un determinado autor posee en la estructura es lo que posibilitará y orientará las estrategias de los artistas para posicionarse en el espacio. De esta manera, llegamos a una definición significativa de la perspectiva bourdieusiana: no es el artista, sino el campo el que detenta los elementos que posibilitan al artista ser lo que es. La segunda característica, complementaria a la primera, es que quienes son el producto de condiciones históricas, sociales, estarían condicionados por el habitus de clases, ya que poseerían disposiciones y esquemas relacionados con sus trayectorias de vida que conformarían espacios de lo posible y que son definidos en la relación establecida entre el habitus y un campo.

Hemos planteado la necesidad de usar categorías propias de nuestros contextos sociales para analizar los fenómenos sociales y culturales. Sin embargo, el concepto de campo artístico resulta clave para reconocer la razón por la cual, de manera general, los actores del campo intelectual latinoamericano no consideran a la literatura marginal contemporánea como literatura legítima. En ese sentido encontramos críticas que la consideran como una forma de manifestación, de declaración, incluso de "subliteratura". Esto ocurre porque quienes forman parte de la élite letrada, que se posiciona como un terreno homogéneo de privilegio, no reconocen en los autores de la literatura marginal contemporánea las mismas características que ven en la literatura canónica o mainstream. La realidad descripta por los escritores de la literatura marginal contemporánea está atrapada por el poder simbólico. Por ende, sus autores son relegados a un papel secundario en la vida social y excluidos del campo intelectual. A su vez, en el campo intelectual el poder simbólico se hace presente y desnuda la efectividad del habitus de una clase respecto de su posición.

El paradigma bourdieusiano posee el gran mérito de identificar la influencia de la ideología y del poder simbólico que impide, o al menos dificulta, el reconocimiento de las obras de autores marginales como literatura, tanto por parte de los especialistas como por parte de la sociedad en general. Asimismo, este paradigma es muy potente al explicar por qué los comportamientos de las clases populares son, en muchas oportunidades, adaptaciones a un sistema que los excluye. Sin embargo, el límite de su perspectiva es su propia noción de habitus y consecuentemente del gusto legítimo. Al buscar identificar el nivel de capital simbólico de cada uno de los distintos grupos sociales, escapa de su vista el hecho de que no hay una homogeneidad absoluta de estos, ya que existe siempre algo, alguien, o un conjunto de individuos que se resisten a ser dominados o clasificados. No obstante, debemos estar atentos para no caer en la trampa de las ideas fuera de lugar, y reafirmar así la relevancia de poder pensar nuestras realidades con nuestras propias categorías, pues el postulado bourdieusiano ha sido desarrollado para una sociedad altamente estructurada como es la francesa de 1970.

Después de esa sucinta descripción de las características del concepto de Campo, para entender algunas de las razones de la resistencia que sufre la literatura marginal contemporánea desde adentro, es inevitable que abordemos las 
diferentes interpretaciones que giran en torno al concepto de cultura, en especial las dos nociones clásicas y a nuestro entender insuficientes: las legitimistas y las miserabilistas.

\subsubsection{Representar lo popular}

En el campo de las ciencias sociales hay diversas teorías que buscan definir conceptualmente la cultura popular. Por un lado, tenemos a la denominada legitimista que posee como a uno de sus representantes a Pierre Bourdieu, a quien hemos estado citando. En su clásico libro La Distinción, publicado en 1979, las clases populares están condenadas a la subordinación a la cultura dominante. Las acciones de los actores subalternos se desarrollan en el conjunto de la formación social que les sería asignada por la clase dominante. La cultura popular sería la contracara de la cultura dominante, el resultado de apropiación desigual de los bienes simbólicos y económicos. Así, posee una cultura propia; la única cultura legítima sería aquella de la clase dominante encasillada al lugar que le es asignado en la reproducción social. En esa concepción, la clase social es definida teniendo en cuenta las relaciones de producción, pero además al habitus de cada clase respecto a su posición. Desde esta perspectiva legitimista, una literatura villera sería imposible.

Según Halbwachs (1970) - perteneciente a la misma corriente teórica- las clases populares buscan, en primer lugar, superar sus necesidades biológicas elementales y primarias, y solo después se interesan por productos de consumo, asociados a los gustos más comunes. En ese sentido, las acciones de la clase dominada estarían invariablemente permeadas por la necesidad. Frente a la mirada legitimista, la cultura popular es inexistente. La dominación simbólica de la clase dominante es total, reproduciendo en el plano cultural la subordinación que existe en el espacio social. En ese contexto, el subalterno no tiene autonomía de acción y tampoco capacidad creadora. Su única función social es la del espectador, reproduciendo los bienes simbólicos y cumpliendo con el rol asignado por la clase dominante. Es por esa razón que, en la teoría legitimista, las obras producidas en las zonas marginales no pueden ser denominadas como literatura. Un problema significativo de esa perspectiva es negar la existencia de la cultura popular. La cultura pertenece a toda la sociedad, es interiorizada por intermedio del habitus. Esa fórmula, desarrollada en un mercado simbólico unificado, no se aplica de forma integral a las sociedades argentinas y brasileñas donde no hay una unificación cultural semejante.

Otra clave analítica que permite estudiar la cultura popular es la noción esencialista que tiene la costumbre de romantizarla. Así, toda la producción y el comportamiento de quienes viven o crean en la marginalidad son consideradas como "auténtica" o "verdadera", en oposición a lo "artificial" de las culturas dominantes. Una crítica inevitable a esa perspectiva es consecuencia de la afirmación de que hay una esencia homogénea presente en todas las culturas populares. Asimismo, otro problema intrínseco a esa perspectiva que identifica una homogeneidad en toda cultura popular frente a nuestro objeto, es el de imposibilitar la identificación de las diferencias idiosincráticas que la literatura marginal asume en cada ciudad y país.

A partir de esa breve exposición, destacamos que tanto los análisis legitimistas como los relativistas poseen límites que no logran dar cuenta de las características de la literatura marginal contemporánea de Argentina y Brasil. Para entender sus características reales no podemos olvidar que lo popular es formado por un conjunto de actores heterogéneos. De esta forma, en la búsqueda por comprender esta literatura y no quedarnos anclados en el binomio legitimista y relativista, es imperioso recordar que lo popular, como hemos dicho anteriormente, no es un conjunto homogéneo que pueda ser clasificado y etiquetado. Además, es importante analizar el contexto en el que emerge la producción: los barrios de las grandes urbes que históricamente son marginalizados, interpretados como zonas peligrosas y pobres y, por ende, sin mucho para ofrecer a la sociedad. 
La literatura, objeto de nuestro estudio, pone no solo la villa o la favela como un espacio social activo en el campo de la literatura, sino también da voces a yoes silenciados que expresan sus concepciones de la realidad. Ese es un punto importante que nos lleva a una primera definición: la literatura marginal es un fenómeno urbano que surge en las villas, que interpela desde la frontera a la sociedad, y que se constituye como un lugar activo conformando, en definitiva, una literatura híbrida.

\section{La hibridación de la literatura}

\subsection{Marginalidad: La villa como frontera activa}

Muchos intelectuales latinoamericanos, como Beatriz Sarlo en su libro La ciudad vista (2009), parten de una perspectiva legitimista para describir las villas como espacios de pobreza en que sus habitantes están condenados a vivir en un permanente contexto de exclusión en sus casas precarias e inconclusas. De esa forma, las familias que viven en esas zonas son descritas como pura necesidad. El futuro para ellos estaría conformado por eventos imprevistos. La vida es interpretada como una lucha por la sobrevivencia que inevitablemente consume todo el tiempo presente y complica el futuro. Estos barrios serían, según esta mirada, un lugar marcado por cierta animosidad, donde lo urbano no es un elemento estable y, según lo que Sarlo denomina la intimidad intima, es sustituida por la intimidad pública (Sarlo, 2009: 79) En esa concepción anclada en la contienda por la sobrevivencia y por un futuro en el que la única certeza es la exclusión y la marginalidad, se hace imposible un espacio en el que surjan escritores y se consolide un campo literario.

Si nos alejamos de esa mirada sesgada que destaca una estigmatización y una exclusión de los intelectuales marginales, podemos vislumbrar otra villa donde el ser fronterizo y villero abre un gran universo de posibilidades.

"Marginal" es un adjetivo que representa indudablemente un espacio creciente en la literatura latinoamericana. Por un lado, porque es la denominación que los propios autores utilizan para denominar sus producciones. En Brasil ha sido publicado por la revista Caro Amigos / Literatura Marginal: A cultura da periferia, ediciones especiales en 2001, 2002 y 2004 que presentaba el trabajo de cuarenta y ocho escritores marginales. Por otro, porque es un adjetivo clave para descifrar el carácter fronterizo. Pero, además, usamos la denominación de villera para acentuar el origen y la procedencia de obras y autores. Esta literatura es marginal y villera. La villa como actor central en la producción de esta literatura. Es desde los barrios que esta literatura se hace visible $y$, consecuentemente, transforma las villas en perceptibles de un modo activo. Esa visibilidad que denominaremos activa es muy distinta de aquella peligrosidad y fealdad que les suele ser asignada; esa forma de analizarla es inversa a aquella que concibe a los barrios desde una perspectiva negativa, como lugar de pobreza y criminalidad. Así, uno de los rasgos principales de la literatura marginal contemporánea es posicionar la villa como un actor central en la vida social que, desde la frontera, interpela a la sociedad y cuestiona el lugar de estigmatización que le suele ser asignado, utilizando el arte de la escritura para crear una literatura propia.

En la definición de Néstor García Canclini (2013: 50), los procesos de hibridación deben ser entendidos como procesos socioculturales que son estructuras y prácticas discretas que se combinan y, al mezclarse, generan nuevas estructuras, nuevas prácticas y nuevos objetos. Ocurren siempre en condiciones históricas y sociales específicas. La fortaleza de ese concepto para nuestro objeto de estudio es posibilitar el análisis en conjunto de lo culto y lo popular que, como vimos, suelen ser analizados de modo separado y así no logran dar cuenta de las características inherentes a esa literatura. Esta perspectiva nos permite salir de los análisis que conciben lo "popular" y lo "culto" como bloques homogéneos. Además, y esto es fundamental, destaca que las identidades no son rasgos fijos $\mathrm{y}$, en consecuencia, permiten la interacción de diversas identidades en un determinando contexto social. 
La literatura marginal es una literatura híbrida y, al mismo tiempo, de frontera, al utilizar la escritura y los géneros canónicos transformándolos, adaptándolos y utilizándolos de modos distintos. El uso de rasgos de oralidad es constante en esta literatura. Para seguir a Canclini, podemos decir que son estrategias de reconversión; o sea, son estrategias que reconvierten el saber de la escritura y lo insertan en nuevas condiciones de producción. En este punto, podemos establecer una segunda definición: los escritores de la denominada literatura marginal crean nuevos usos y recursos al arte de la escritura.

Al irrumpir desde los márgenes del mundo social y de la sociedad letrada, en las obras están presentes la persistente tensión entre las estructuras cultas típicas de las producciones literarias y los mecanismos propios de la idiosincrasia de esa producción. El lenguaje cumple una función primordial en la literatura marginal villera, y su potencia se desnuda, por ejemplo, en el uso del lunfardo y de los errores gramaticales como disrupción lingüística que responde a la homogénea superficie de la literatura canónica. Además del lenguaje, las tramas de las novelas destacan su particularidad de producción y narración, reflejando la vida en los barrios con sus problemáticas, con sus dilemas, con sus momentos de diversión. Al reivindicar su lenguaje y su lugar de producción, la literatura marginal enarbola su particularidad de presentar la voz de los subalternos, de los invisibles sociales, para utilizar la denominación de Jessé de Souza (2009: 14), visibilizando y empoderando a la villa como lugar de producción activo, rompiendo con una lógica en la cual era interpretada como un lugar de subordinación.

La literatura marginal contemporánea posee diversas características propias si la comparamos con el campo literario canónico, y presenta dos características definitorias: por un lado, destaca y acepta el dilema entre visibilización e invisibilización al dar voces a personajes marginales y al poner en el centro de sus narrativas a las villas. Por otro lado, al ser una literatura híbrida, solamente puede surgir en ese contexto "marginal" urbano, fronterizo con la sociedad.

\subsection{La escritura y la oralidad}

En la lectura que hace Terry Eagleton de Bajtin, la tensión en las novelas ocurre cuando los discursos, como por ejemplo los de clase, se chocan y se confunden. Los signos no son elementos estáticos sino dialógicos. En esa clave analítica, los discursos pertenecen a la frontera. Según Eagleton (1986: 135) son un lenguaje centrífugo que fractura los códigos autorizados. Consecuentemente, en las novelas no entra en juego solo el significado de quien habla, sino también bajo qué condición lo hace.

Los autores de las clases populares, marginales, subalternos, describen en sus obras literarias la vida cotidiana de su realidad social. Retratan los problemas y también los mecanismos de sociabilidad cultural, los aspectos de convivencia en los barrios que habitan. Para eso, subvierten la norma culta de escritura y, al utilizar su lenguaje, ejercitan una forma de resistencia pasiva. La estructura narrativa de las novelas producidas por los autores de la literatura marginal contemporánea y su modo de describir la realidad son formas de resistencia a la falta de reconocimiento social, a la exclusión sistemática, en un contexto de naturalización de la desigualdad. Nelly Richard (2002) denomina a este tipo de narrativa como narrativa de residuos, dado que utiliza géneros que son herramientas por excelencia de la cultura ortodoxa y los subvierten para hacer visibles un conjunto de yoes que estaban condenados a ser invisibles, o solo visibles en los noticieros policiales.

Desde la sociología podemos interpretar tanto esta práctica de escritura como las desconfianzas que surgen respecto de ella en el campo literario, utilizando el binomio tácticas / estrategias de Michel De Certeau (1999:100). Así, podemos decir que el poder simbólico utiliza un conjunto de estrategias para silenciar, invisibilizar a los habitantes de los barrios humildes. Por otro lado, estos desarrollan un conjunto de tácticas que, a diferencia de las estrategias, no son organizadas, sino que surgen como respuesta al poder hegemónico. 
Sus herramientas de actuación están basadas en las experiencias cotidianas, en su cultura. En este sentido, el lunfardo, el hip-hop y la cumbia son ejemplos de esas experiencias denominadas por De Certeau como repositorio de cultura, marcas identitarias que aparecen en las narrativas de la literatura marginal contemporánea de Argentina y Brasil.

\section{Conclusión}

A lo largo de este recorrido teórico hemos podido identificar un nuevo espacio en la narrativa contemporánea latinoamericana que inaugura un lugar de resistencia desde lo cultural y lo lingüístico, y que presenta estrategias discursivas de ubicación en la literatura marginal contemporánea de Argentina y Brasil. La disrupción lingüística, la pluralidad enunciativa como marca cultural de referencia, la presencia viva de elementos populares que dan cuenta de voces por contarse, de nombres por oir, de vidas por narrar, son el eje central de esta nueva literatura que comenzamos a explorar en este artículo, pero que supondrá muchas palabras aún en un futuro más plural y más heterogéneo para Latinoamérica.

\section{Referencias}

Becker, Howard. 2015. Para hablar de la sociedad la sociología no basta. Buenos Aires: Siglo XXI Editores.
Bourdieu, Pierre. 2010. El sentido social del gusto: Elementos para una sociología de la cultura. Buenos Aires, Siglo XXI Editores.

Bourdieu, Pierre. 1998. La Distinción: Criterio y bases sociales del gusto. Buenos Aires: Taurus.

Canclini, Néstor García. 2013. Culturas hibridas: Estrategias para entrar y salir de la modernidad. Buenos Aires: Paidós.

De Certeau, Michel. 1998. A invenção do cotidiano, Petrópolis: Editora Vozes.

Eagleton, Terry. 1986. A contrapelo. Buenos Aires: Nueva Visión.

Halbwachs, Maurice. 1970. La clase ouvrière et les niveaux de vie. Paris: Gordon \& Breach.

Richard, Nelly. 2002. Intervenções críticas: arte, cultura, gênero e política. Belo Horizonte: Editora UFMG.

Sarlo, Beatriz. 2009. La ciudad vista: Mercancías y cultura urbana. Buenos Aires: Siglo XXI Editores.

Souza, Jessé. 2009. A ralé brasileira: Quem é e como vive. Belo Horizonte: UFMG. 
